\title{
Syndrome de Melkersson-Rosenthal à caractère familial : à propos d'un cas
}

\author{
Melkersson-Rosenthal syndrome with family characters: a case report
}

\author{
HAJER HENTATI ${ }^{1}$, SONIA HAMMAMI ${ }^{2}$, LAMIA OUALHA ${ }^{1}$, IMÈNE MANNA ${ }^{1}{ }^{1}$, JAMIL SELMI ${ }^{1}$
}

\begin{abstract}
RÉSUMÉ
Un patient âgé de 18 ans consulte pour une macrochéilite supérieure évoluant par poussées sans que la lèvre reprenne son aspect normal entre les poussées. L'anamnèse révèle un antécédent de paralysie faciale périphérique gauche. L'examen endobuccal montre entre autres une langue plicaturée et un œdème gingival. Devant cette triade symptomatique, le diagnostic clinique du syndrome de Melkersson-Rosenthal a été établi. La récidive de l'œdème a soulevé des difficultés thérapeutiques. L'enquête auprès de la famille du patient a permis d'établir l'arbre généalogique permettant de retrouver ces atteintes chez certains membres de cette famille. Med Buccale Chir Buccale 2007; 13 : 29-34.
\end{abstract}

mots clés : syndrome de Melkersson-Rosenthal, paralysie faciale, œdème, granulomatose.

\section{SUMMARY}

A case of Melkersson-Rosenthal syndrome in an 18 years old male patient with family characters is reported. The patient consults with a recurring swelling of the upper lip. A transient facial nerve palsy of the left side of the face was preceded the oedema. Intraoral examination revealed a fissured tongue and a gingival enlargement. In view of the presence of the classical triad of signs (facial swelling, facial paralysis and fissured tongue), the clinical diagnosis of Melkersson-Rosenthal syndrome was made. The family history showed that some members were presented facial swelling and/or facial paralysis; the family tree is traced. Treatment used systemic corticosteroids and intralesional injection of triamcinolone acetonide. Med Buccale Chir Buccale 2007; 13 : 29-34.

key words: Melkersson Rosenthal syndrome, facial palsy, edema, granulomatosis.

\section{REMERCIEMENTS}

Nous remercions le Professeur Abd Ellatif Zakhama, Chef du Service d'Anatomie et de Cytologie pathologiques, Hôpital universitaire Fattouma Bourguiba de Monastir pour l'iconographie anatomo-pathologique.

1. Service de Médecine et Chirurgie buccales Clinique hospitalo-universitaire d'Odontologie Monastir Tunisie

2. Service de Médecine interne Centre hospitalo-universitaire Fattouma Bourguiba Monastir Tunisie

Demande de tirés à part:

Hajer Hentati-Aloulou Clinique hospitalo-universitaire d'Odontologie de Monastir Service de Médecine et Chirurgie buccales Avenue Avicenne 5019 Monastir Tunisie hajer.hentati@laposte.net ou hajer.hentati@fmdm.rnu.tn 
Le syndrome de Melkersson-Rosenthal (SMR) est une entité rare, définie par la triade œdème facial, paralysie faciale et langue plicaturée. Ce travail a pour but de décrire un nouveau cas de SMR et d'en discuter le caractère familial car il a été retrouvé chez d'autres membres de la famille.

\section{OBSERVATION}

II s'agit d'un patient âgé de 18 ans qui consulte pour une macrochéilite supérieure évoluant par poussées depuis cinq mois sans que la lèvre reprenne son aspect normal entre les poussées (Fig. 1). L'anamnèse révèle que ce patient a présenté une paralysie faciale périphérique (PFP) gauche ayant précédé de six mois l'œdème labial et ayant régressé sous traitement médical sans laisser de séquelles. L'anamnèse familiale permet de retrou- médecine buccale chirurgie buccale

VOL. $14, \mathrm{~N}^{\circ} 1$ 2008

page 30 ver des cas de PFP et d'œdème labial chez certains membres de sa famille. L'examen clinique montre la présence d'un œdème de la lèvre supérieure et de la gencive, d'une langue plicaturée (Fig. 2) et l'absence de foyers infectieux dentaires. La présence de la triade PFP, œdème facial et langue plicaturée oriente vers le diagnostic de SMR. Une biopsie des glandes salivaires accessoires a été réalisée; l'examen anatomo-pathologique montre la présence d'un discret infiltrat lymphocytaire et l'absence de granulomes épithélioïdes (Fig. 3). La prescription de prednisone à doses dégressives pendant un mois entraine une régression de l'œdème. Quatre mois plus tard, le patient revient avec une récidive de la macrochéilite; il est hospitalisé dans

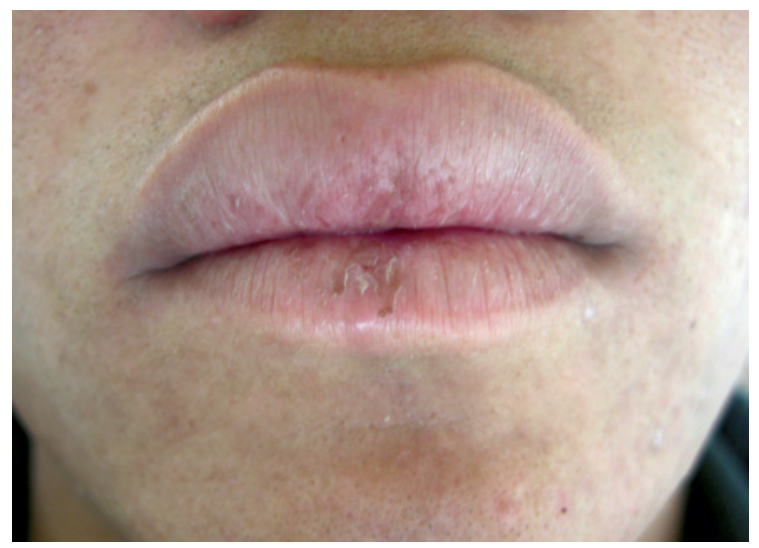

Figure 1 : Tuméfaction de la lèvre supérieure. Upper lip swelling. un service de médecine interne pour réaliser différentes investigations. Les examens biologiques (vitesse de sédimentation, CRP, taux du complément C2 et C4 et bilan thyroïdien) sont normaux ; l'examen neurologique ne montre aucun déficit moteur ou sensitif ; la tomodensitométrie cérébrale et la colonoscopie ne trouvent aucune anomalie. L'examen anatomo-pathologique de la deuxième biopsie de la lèvre, plus profonde que la première, conclut à l'absence de granulomes épithélioïdes. La corticothérapie (prednisone), reprise à la dose de $0,5 \mathrm{mg} / \mathrm{kg} / \mathrm{j}$ fait régresser l'œdème; cependant, une récidive est survenue lors de la réduction de la dose à $0,2 \mathrm{mg} / \mathrm{kg} / \mathrm{j}$. Un autre protocole thérapeutique est alors adopté : injection mensuelle intralésionnelle de $40 \mathrm{mg}$ ( $1 \mathrm{ml}$ ) de triamcinolone acétonide (Kénacort Retard ${ }^{\circledR}$ ) en trois points dans la lèvre supérieure. Les résultats sont satisfaisants avec un recul d'un an.

Concernant le caractère familial du SMR, le déplacement d'une équipe médicale auprès de treize membres de la famille permet de reprendre l'anamnèse et l'examen clinique, et de recueillir également des informations concernant six autres membres de la famille. Avec l'ensemble des données, on réalise un arbre généalogique présenté selon Muller et coll. [1] (Fig. 4). Pour la première et la deuxième générations, aucune information sûre n'a pu être rapportée. Pour la troisième et la quatrième générations, 19 membres de la famille présentent au moins un symptôme de la triade syndromique dont trois avec deux symptômes et trois avec le syndrome complet (le cas du patient rapporté compris).

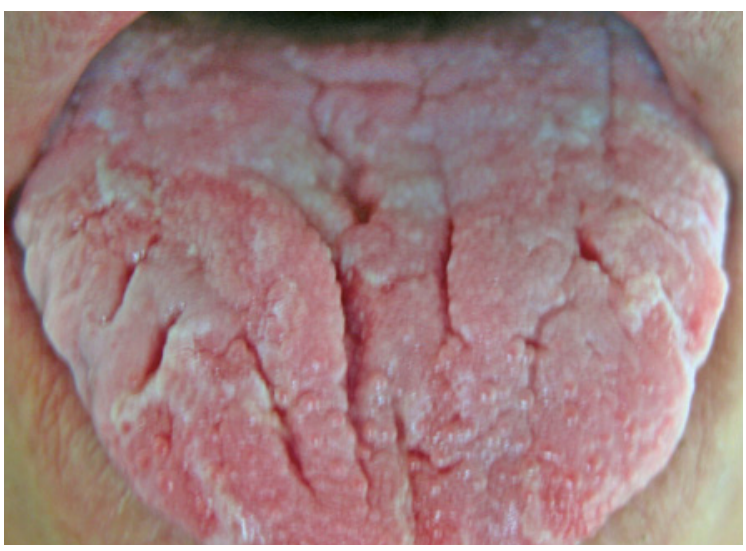

Figure 2 : Langue plicaturée. Fissured tongue. 


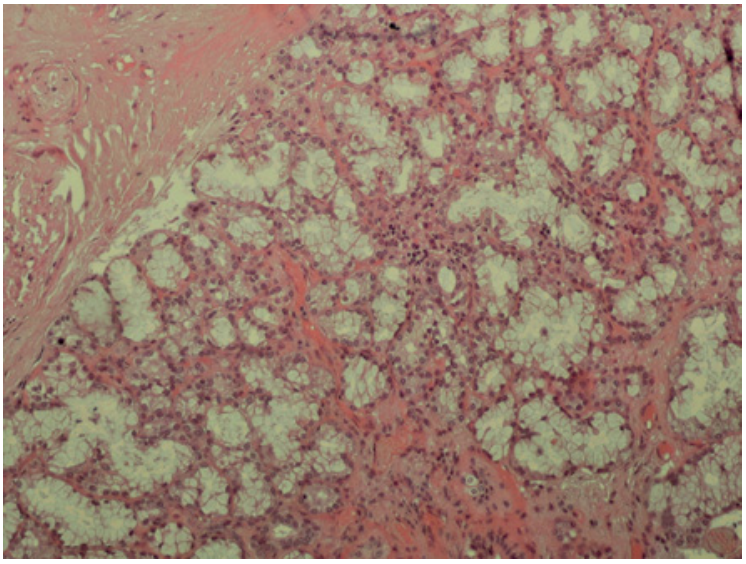

Figure 3 : Discret infiltrat lymphocytaire des glandes salivaires accessoires (H\&E, x 100).

Discret lymphocytic infiltrate of the minor salivary glands (H\&E, $x$ 100).

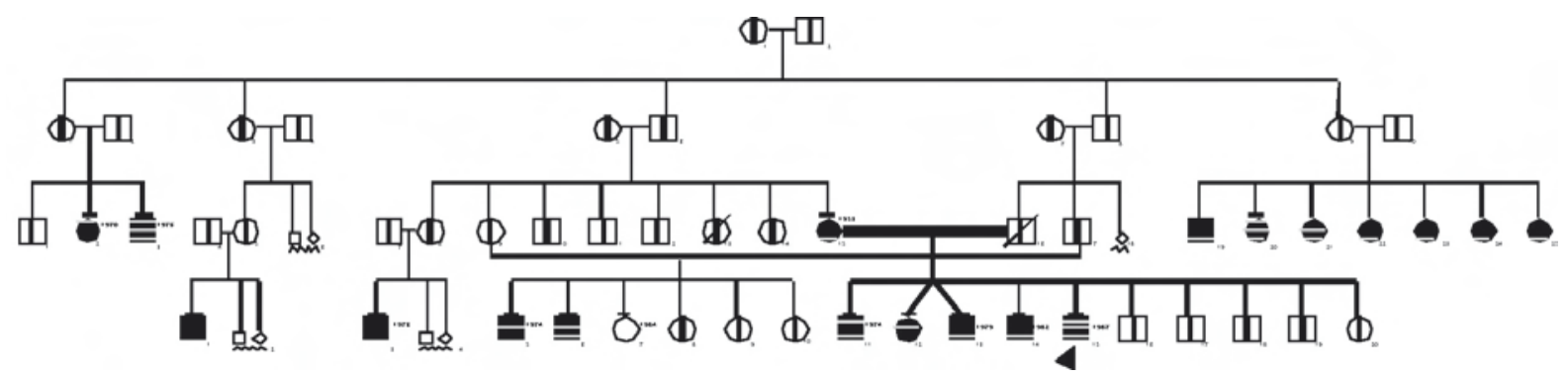

Figure 4 : Arbre généalogique.

Family tree.

\section{COMMENTAIRES}

médecine

buccale

chirurgie

buccale

VOL. $14, \mathrm{~N}^{\circ} 1$

2008

Paralysie faciale

En 1928, Melkersson décrit l'association d'une

CEdème faciale

paralysie faciale et d'un œdème oro-facial. En 1931, Rosenthal complète le tableau clinique par

Langue olicaturée

la description d'une langue plicaturée. C'est un syndrome rare, son incidence est estimée à $0,08 \%$ de la population ${ }^{22]}$. Cette affection débute surtout dans la deuxième décade mais peut survenir à tout âge, même chez de jeunes enfants [3]. L'âge des 42 patients présentés dans l'étude de Homme Femme Zimmer et coll. [4] varie de 6 à 73 ans avec une atteinte féminine plus importante; pour d'autres auteurs, il n'y a aucune prédilection de sexe [2]. La plupart des SMR sont incomplets; selon Stosiek et coll. seuls $8 \%$ des cas présentent un syndrome complet [5].

Sur le plan clinique, l'œdème facial constitue souvent le symptôme inaugural $(81 \%$ des cas pour Zimmer et coll. [4], 82 \% des cas pour Stosiek et coll. [5]); il prédomine sur les lèvres, intéresse le plus souvent la lèvre supérieure, parfois les deux lèvres de façon asymétrique [6]. L'ensemble de la 
médecine

buccale

chirurgie

buccale

VOL. $14, \mathrm{~N}^{\circ} 1$ 2008

page 32 muqueuse buccale peut être touchée par un œdème : le palais, les gencives et, plus rarement, le plancher buccal et la langue [2]. Worsaae et Pindborg ${ }^{[7]}$ trouvent une atteinte gingivale récidivante chez 13 patients sur 30 présentant un SMR. L'œdème peut avoir d'autres localisations faciales (joues, nez, paupières, front...), extrafaciales ou même extracéphaliques ${ }^{[8]}$. L'œdème de consistance molle peut évoluer vers la fibrose et prend alors la consistance d'un œdème solide [9]. Pour le cas rapporté, l'œdème intéresse la lèvre supérieure et la gencive. Neuf membres de la famille ont présenté au moins une poussée de macrochéilite qui constitue le symptôme unique pour quatre d'entre eux. L'âge de survenue minimal est de 10 ans.

La paralysie faciale est de type périphérique, partielle ou complète, uni ou bilatérale, à début brusque ou progressif, évoluant par poussées avec une régression après deux ou trois semaines $[6,8]$. Elle est inaugurale dans $8{ }^{[5]}$ à $39 \%{ }^{[8]}$ et s'observe chez $30{ }^{[10]}$ à $47 \%{ }^{[8]}$ des patients atteints. Khandpur et coll. ${ }^{[11]}$ ont décrit un cas de SMR avec une paralysie touchant plusieurs nerfs crâniens. La PFP est présente chez cinq membres de la famille dont trois où la triade syndromique est complète. Le membre III3 (Fig. 4) qui a été le plus jeune sujet atteint (première poussée à l'âge de 14 ans) est le seul à avoir présenté trois poussées.

La langue plicaturée constitue le deuxième signe clinique par ordre de fréquence : elle est observée chez plus de $40 \%$ des patients [11]. La langue a un aspect épaissi, élargi avec des sillons profonds ${ }^{[6]}$. Ce signe est de moins en moins considéré comme un symptôme majeur de la triade classique, mais plutôt comme un signe en faveur du SMR [5]. Cet aspect est retrouvé chez 10\% de la population [12]. Dans la famille étudiée, 14 personnes sur 20 présentent une langue plicaturée dont 8 cas isolés qui ne peuvent donc pas être reliés formellement au SMR.

Des signes atypiques sont parfois présents : atteinte d'autres nerfs crâniens (III, V, XII), migraines et signes psychiatriques [3].

L'examen anatomo-pathologique constitue le seul examen paraclinique qui soit contributif. Deux types histologiques ont été décrits: le type granulomateux avec des granulomes à cellules épi- thélioïdes, non caséeux, prédominant autour des vaisseaux et constitués de cellules inflammatoires mononucléées, et un type nodulaire lympho-plasmocytaire caractérisé par des nodules riches en lymphocytes, bordés d'une couronne de plasmocytes et d'histiocytes ${ }^{[2,3]}$. Le premier type est considéré comme spécifique du SMR mais il ne suffit pas à déterminer le diagnostic qui reste clinique ; il est cependant très contributif dans les formes monosymptomatiques. En contrepartie, l'absence d'un infiltrat granulomateux ne permet pas d'éliminer le diagnostic de SMR ${ }^{[4]}$ comme dans le cas rapporté. Hornstein et coll. [13] ont proposé une classification basée sur des signes majeurs et des signes mineurs. Les signes majeurs, dits de premier ordre, comprennent la PFP, l'œdème facial et la présence de granulomes épithélioïdes; les signes mineurs, dits de deuxième ou de troisième ordres, un dysfonctionnement pouvant intéresser d'autres nerfs crâniens moteurs ou sensitifs et des perturbations de la régulation neurovégétative. La découverte de symptômes mineurs concomitants s'avère être d'une grande utilité pour la reconnaissance des formes incomplètes de SMR.

L'étiologie du syndrome demeure inconnue. II pourrait s'agir d'un dérèglement du système nerveux, d'une origine allergique ou d'une origine infectieuse ${ }^{[6,10]}$. Apaydin et coll. ${ }^{[14]}$ suggèrent une possible implication du Mycobacterium tuberculosis. Smeets et coll. [15] ont mis en évidence une translocation génétique chez une patiente atteinte d'un SMR. Le caractère familial a été mentionné dans certaines publications; il s'agit d'observations de cas de SMR survenant simultanément chez plusieurs membres d'une même famille ou apparaissant dans une même famille sur plusieurs générations successives ${ }^{[8,16]}$. Ces deux aspects existent dans le cas rapporté puisqu'on retrouve le syndrome complet dans les générations III et IV et des paralysies faciales survenues simultanément en 1990 chez quatre membres de la famille (III2, III3, III20, et IV11).

Le diagnostic différentiel se pose avec la maladie de Crohn, la sarcoïdose et certaines infections spécifiques en particulier la tuberculose. La maladie de Crohn, maladie inflammatoire chronique de l'intestin, se manifeste habituellement par des 
crampes abdominales récurrentes et des diarrhées chroniques ${ }^{[17]}$. Pour la sarcoïdose, la radiographie pulmonaire et le dosage de l'enzyme de conversion de l'angiotensine permettent d'orienter le diagnostic [18]. Quant au diagnostic de la tuberculose, l'intra-dermoréaction à la tuberculine et la radiographie pulmonaire sont des examens complémentaires capitaux [9].

La macrochéilite de Miesher est considérée, par la plupart des auteurs, comme une forme monosymptomatique de SMR ${ }^{[12]}$. Wiesenfeld et coll. [18] ont introduit, en 1985, le concept de granulomatose oro-faciale englobant le SMR et la macrochéilite de Miesher.

Sur le plan thérapeutique, c'est l'œdème facial qui soulève le plus de difficultés. La corticothérapie systémique ou locale (injections intralé-

\section{RÉFÉRENCES}

1 - Müller H, Imhasly P, Leuthold M. Le diagnostic des maladies héréditaires. Schweiz Med Forum $2005 ; 5: 611-4$.

2 - De Labrouhe C, Favre-Dauvergne E, Divaris M, Vaillant J M. Actualités thérapeutiques de la macrochélite dans le syndrome de MelkerssonRosenthal : à propos d'un cas. Rev Stomatol Chir Maxillofac 1994 ; 95 : 190-2.

3 - Spielmann A-C, Maury F, George J-L. Syndrome de Melkersson-Rosenthal. Concepts anatomocliniques et évolutifs. J Fr Ophtalmol 2000 ; 23 : 261-4.

4-Zimmer WM, Rogers III RS, Reeve CM, Sheridan PJ. Orofacial manifestations of Melkersson-Rosenthal syndrome. A study of 42 patients and review of 220 cases from the literature. Oral Surg Oral Med Oral Pathol 1992 ; $74: 610-9$.

5 -Stosiek N, Birolleau S, Capesius C, Hornstein OP. Chronicité et incertitudes diagnostiques du syndrome de MelkerssonRosenthal. Analyse des modalités évolutives de cinq cas. Ann Dermatol Venereol 1992 ; 119 : 635-8.

6 - Piette E. Pathologie des lèvres (pp 865-911). In Traité de pathologies buccale et maxillo-faciale. Piette E, Reychler H ed : De Boeck-Université, Bruxelles, 1991. sionnelles) représente le traitement de choix ; elle contribue à la régression des symptômes mais elle n'empêche pas les récidives [12]. D'autres molécules ont été utilisées mais aucune n'a prouvé son efficacité (clofazimine, minocycline, lymécycline, métronidazole, antihistaminiques, danazol, hydroxychloroquine, méthotrexate et thalidomide) $[9,19]$.

La chirurgie de résection peut être indiquée dans les formes récidivantes ou fibrosantes de macrochéilite ; selon Camacho et coll. [20], elle devrait être suivie d'injections intralésionnelles de corticoïdes. Le traitement de la paralysie faciale est essentiellement médical ; la décompression du nerf facial dans son segment intrapétreux est exceptionnellement indiquée. La langue plicaturée est le plus souvent bien tolérée ${ }^{[2]}$.

7 - Worsaae N, Pindborg JJ. Granulomatous gingival manifestations of Melkersson-Rosenthal syndrome. Oral Surg Oral Med Oral Pathol $1980 ; 49: 131-8$.

8 -Grosshans E, Pfeffer S. Le syndrome de Melkersson-Rosenthal. La macrochéilite granulomateuse de Miescher. Ann Dermatol Venereol 1991; $118: 245-51$.

9 - Kauzman A, Quesnel-Mercier A, Lalonde B. Granulomatose orofaciale : 2 rapports de cas et recensement de la littérature. J Can Dent Assoc 2006 ; 72 : 325-9.

10 - Bataineh AB, Pillai KG, Mansour M, Abu Al-Khai A. An unusual case of the Melkersson-Rosanthal syndrome. A case report. Oral Surg Oral Med Oral Pathol Oral Radiol Endod 1995 ; 80 : 289-92.

11 - Khandpur S, Malhotra AK, Khanna N. Melkersson-Rosenthal syndrome with diffuse facial swelling and multiple cranial nerve palsies. J Dermatol 2006 ; $33:$ 411-4.

12 - El-Hakim M, Chauvin P. Orofacial granulomatosis presenting as persistent lip swelling: review of 6 new cases. J Oral Maxillofac Surg 2004 ; $62: 1114-7$.

13 - Hornstein OP, Stosiek N, Schonberger A, Meisel-Stosiek M. Klassifikation und klinische Variationsbreite des Melkersson-RosenthalSyndroms (MRS). Z Hautkr 1987 ; 62 : 1471-5. médecine

buccale

chirurgie

buccale

VOL. $14, \mathrm{~N}^{\circ} 1$

2008

page 33 
14 - Apaydin R, Bahadir S, Kakklikkaya N, Bilen N, Bayramgürler D. Possible role of Mycobacterium tuberculosis complex in Melkersson-Rosenthal syndrome demonstrated with Gen-Probe amplified Mycobacterium tuberculosis direct test. Aust J Dermatol 2004 ; 45 : 94-9.

15 - Smeets E, Fryns JP, Van den Berghe $H$. Melkersson-Rosenthal syndrome and de novo autosomal $\mathrm{t}(9 ; 21)(\mathrm{p} 11 ; \mathrm{p} 11)$ translocation. Clin Genet 1994 ; 45 : 323-4.

16 - Chaine P, Woimant F, Lemay V, Haguenau M. Syndrome de Melkersson-Rosenthal à caractère familial. Presse Med $1990 ; 19: 33$.

17 - Alawi F. Granulomatous diseases of the oral tissues: differential diagnosis and update. Dent Clin N Am 2005 ; 49 : 203-21.
18 - Wiesenfeld D, Ferguson MM, Mitchell DN, MacDonald DG, Scully C, Cochran K, Russell RI. Oro-facial granulomatosis. A clinical and pathological analysis. Q J Med 1985 ; 54 : 101 13.

19 - Pigozzi B, Fortina AB, Peserico A. Successful treatment of Melkersson-Rosenthal syndrome with lymecycline. Eur J Dermatol 2004 ; 3 : 1667.

20 - Camacho F, Garcia-Bravo B, Carrizosa A. Treatment of Miescher's cheilitis granulomatosa in Melkersson-Rosenthal syndrome. J. Eur Acad Dermatol Venerol 2001 ; 15 : 546-9.

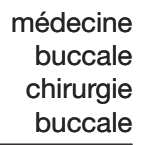

VOL. $14, \mathrm{~N}^{\circ} 1$ 2008 